Proceedings of the 31st Annual Meeting of the Brazilian Embryo Technology Society (SBTE); Cabo de Santo Agostinho, PE, Brazil, August 17th to 19th, 2017.

\title{
The role of L-carnitine during oocyte in vitro maturation: essential co-factor?
}

\author{
Kylie R. Dunning ${ }^{1,2,3,4}$, Rebecca L. Robker ${ }^{3}$ \\ ${ }^{1}$ Australian Research Council Centre of Excellence for Nanoscale BioPhotonics, University of Adelaide, Adelaide, SA, 5005, \\ Australia. \\ ${ }^{2}$ Institute for Photonics and Advanced Sensing, University of Adelaide, Adelaide, SA, 5005, Australia. \\ ${ }^{3}$ The Robinson Research Institute, Adelaide Medical School, University of Adelaide, Adelaide, SA, 5005, Australia.
}

\begin{abstract}
In vitro maturation (IVM) of oocytes is a promising technology for both the treatment of human infertility and in animal production as a means of improving genetic gain. However, IVM derived oocytes remain inferior to those matured in vivo with reduced developmental potential. The environment in which an oocyte matures in vitro is vastly different to in vivo where maturation takes place within the ovarian follicle. The in vitro environment differs in oxygen concentration, exposure to light, and metabolite composition of culture media $v s$. follicle fluid, to name a few. Human follicle fluid contains the metabolite Lcarnitine and has shown to be associated with human fertility. L-carnitine has known biological functions as an essential co-factor for beta-oxidation, regulating ATP production from lipids, and as a potent antioxidant. Importantly, it appears that cumulus cells and the oocyte lack the machinery to synthesize L-carnitine de novo. The inability for local production of L-carnitine during IVM and its importance in human fertility warrants investigation of its affects during IVM. The potential to improve oocyte quality by inclusion of L-carnitine in the culture media thus increasing the capacity for betaoxidation and/or antioxidant activity of the culture media is receiving increased attention. This review summarizes studies to date investigating the developmental importance of L-carnitine during IVM and the mechanisms by which improved developmental potential is elicited. Overall, the inclusion of L-carnitine during IVM of several species results in improved oocyte quality with increased development to blastocyst. This is likely due to the antioxidant capacity of L-carnitine and its ability to increase ATP production from intracellular lipid stores.
\end{abstract}

Keywords: antioxidant, beta-oxidation, in vitro maturation, L-carnitine, oocyte,

\section{Introduction}

The final stages of oocyte maturation in vivo occur once folliculogenesis has reached the antral stage. The periovulatory antral follicle is capable of responding to the LH-surge from the pituitary which initiates the ovulatory cascade. The LH-surge acts on the granulosa cells of the follicle which transmit the ovulatory signal to the cumulus oocyte complex (COC) via secretion of EGF-like peptides. This initiates nuclear and cytoplasmic maturation in the oocyte and the cumulus cells to undergo expansion via the production of vast amounts of extracellular matrix. Following this, ovulation ensues with the COC then picked up by the fimbria of the oviduct and transported into the oviduct where fertilization can occur. Following fertilization, meiosis is completed and the one-cell zygote develops to the blastocyst stage whilst transcending through the oviduct to the uterus where the blastocyst embryo implants and pregnancy commences.

The ability of a zygote to successfully complete preimplantation embryo development, implant in the uterus and develop into a healthy offspring is dependent on the quality, or developmental competence, of the oocyte. The environment in which the oocyte matures in vivo is modified by maternal diet and metabolic status, which can negatively affect oocyte quality and the health of the offspring (Lane et al., 2014). Similarly, in vitro maturation of oocytes occurs in a sub-optimal environment drastically different from that which occurs in vivo. For in vitro maturation the $\mathrm{COC}$ is removed from the follicle prior to the LH-surge and the final stages of maturation occur in vitro in the absence of the follicle and follicular fluid.

Oocyte in vitro maturation (IVM) is a potentially useful assisted reproductive technology both clinically and in animal production (Gilchrist and Thompson, 2007). Developmental potential of IVM oocytes following fertilization is however, lower than their in vivo matured counterparts (Rizos et al., 2002; Gilchrist and Thompson, 2007). Oocytes matured in vitro have lower rates of development to the blastocyst stage and have increased rates of miscarriage (Buckett et al., 2008). The direct mechanisms responsible for the poorer oocyte quality are the topic of continued investigation and not as yet, clear. As discussed above, the environment in which the oocyte matures in vitro is dramatically different to in vivo maturation. In vitro maturation differs in the exposure to light, oxygen concentration and different metabolite composition of culture media $v s$. follicular fluid, among many other factors.

The composition of the IVM culture media is known to affect $\mathrm{COC}$ metabolism and metabolic rate (reviewed in Brown et al., 2017). Further, oocyte developmental competence is affected by metabolism with generation of sufficient levels of ATP required for oocyte growth, meiosis, fertilization and the early stages 
of preimplantation embryo development (Van Blerkom et al., 1995). The metabolism of carbohydrates, glucose, pyruvate and lactate for the production of ATP, have been well characterized in the COC (Sutton et al., 2003; Brown et al., 2017). Metabolism of fatty acids via betaoxidation is a potent source of energy, several fold more energy dense than glucose, and the developmental importance of this form of metabolism for the $\mathrm{COC}$ is gaining recognition. The use of oocyte intracellular stores for energy production during IVM is of particular interest in species with high levels of stored lipid such as bovine, sheep and porcine (reviewed in Dunning et al., 2014b). Even in species with comparatively low levels of oocyte lipid (mouse and human; Dunning et al., 2014b), beta-oxidation during oocyte maturation appears essential for subsequent developmental competence (Dunning et al., 2010, 2011, 2014b) Betaoxidation of fatty acids requires the essential co-factor L-carnitine to shuttle activated fatty acids from the cytosol into mitochondria. The addition of L-carnitine to the in vitro culture of cell lines in known to increase beta-oxidation (Nada et al., 1995; Huynh et al., 2014). L-carnitine also acts as a powerful antioxidant shown in other in vitro systems to quench reactive oxygen species (ROS) and in turn protect cells from oxidant injury (Ye et al., 2010). Oocytes matured in vitro are exposed to increased levels of ROS (reviewed in Combelles et al., 2009), thus inclusion of L-carnitine may be of benefit. The role of L-carnitine during IVM has gained interest in recent years as evidenced by a growing body of literature. This review will highlight studies utilizing Lcarnitine during IVM, the resultant impact on oocyte quality and the mechanism(s) by which it acts.

\section{Carnitine and fertility}

Carnitine is a naturally occurring quaternary amine with the L-stereoisomer (3R)-3-hydroxy-4(trimethylazaniumyl)butanoate having potent bioactivity. Synthesis of carnitine from lysine and methionine occurs mainly in the liver with highes levels found in skeletal muscle and heart (Vaz and Wanders, 2002). Dietary sources high in carnitine include meat and dairy (Rebouche, 1992). L-carnitine is essential for the transport of activated long chain fatty acids from the cytoplasm into mitochondria where, via beta-oxidation, ATP can be generated. Carnitine is also responsible for the transfer of peroxisomal betaoxidation metabolites to the mitochondria for completion of oxidation to $\mathrm{CO}_{2}$ and $\mathrm{H}_{2} \mathrm{O}$ via the TCA cycle (reviewed in Vaz and Wanders, 2002). Other functions of carnitine include the maintenance of the acyl-CoA:CoA ratio and as a means of storing cellular energy in the form of acetylcarnitine (Vaz and Wanders, 2002). Additionally, L-carnitine has been used during in vitro culture and animal studies as a scavenger of free radicals, in turn protecting antioxidant enzymes from oxidant injury (Bremer, 1983; Sener et al., 2004; Gulcin, 2006; Kolodziejczyk et al., 2011).

Carnitine is present in human follicular fluid (Dunning and Robker, 2012; Montjean et al., 2012; Valckx et al., 2012; Varnagy et al., 2013; Zhao et al.,
2015; Giorgi et al., 2016). While follicular fluid from these studies was sampled following ovulation induction, we can infer from the chemical properties of carnitine and its presence in serum, that carnitine would be present during oocyte maturation in vivo. In relation to oocyte quality and human fertility, it was shown that decreased carnitine (total and free) and acylcarnitine metabolites in follicular fluid was associated with increased numbers of oocytes $(>9)$ and embryos $(>6)$ from women undergoing IVF (Varnagy et al., 2013). We can speculate that decreased levels of carnitine and acylcarnitines were associated with improved reproductive potential as a result of their increased utilization via beta-oxidation during oocyte maturation (Varnagy et al., 2013). A similar association was not seen in in a separate study, however, only total carnitine was measured and compared with pregnant and nonpregnant cycles (Montjean et al., 2012). Replication of the association between carnitine (total and free) and the acylcarnitine metabolites with oocyte quality and fertility outcomes, in particular live birth, would be of interest.

Current evidence suggests that cumulus cells and oocytes do not have the capacity to biosynthesize Lcarnitine from precursor amino acids as they lack the metabolic machinery (Montjean et al., 2012). This study examined human cumulus cells and oocytes at the end of maturation and only mRNA for two of the enzymes in the biosynthesis pathway. Whether regulation of these genes or the presence of the enzymes themselves occurs earlier in maturation of COCs is yet to be determined.

The potential developmental importance of carnitine and its acylcarnitine derivatives for oocyte quality following in vivo maturation of human oocytes and the apparent inability of COCs to synthesize carnitine points to the importance of investigating the role of carnitine during in vitro maturation. There is a growing number of studies that have investigated the role of L-carnitine during IVM in various species including mouse (Dunning et al., 2010, 2011; Paczkowski et al., 2014), bovine (Ferguson and Leese, 2006; Phongnimitr et al., 2013; Giorgi et al., 2016; Sovernigo et al., 2017), porcine (Hashimoto, 2009; Somfai et al., 2011; Wu et al., 2011; You et al., 2012) and sheep (Reader et al., 2015; Mishra et al., 2016a). The effects of L-carnitine on oocyte quality and the associated mechanism(s) are detailed in the following sections.

\section{Importance of beta-oxidation and its modulation by L-carnitine during IVM}

There is a growing body of literature describing the importance of fatty acid beta-oxidation as a source of energy during oocyte IVM (Ferguson and Leese, 2006; Sturmey et al., 2006; Downs et al., 2009; Dunning et al., 2010; Dunning and Robker, 2012; Valsangkar and Downs, 2013; Dunning et al., 2014b; Paczkowski et al., 2014). This is not surprising when one considers the energy dense nature of fatty acids with the potential to produce 106 ATP molecules from a 
single fatty acid, greater than 3.5 -fold the capacity of a single glucose molecule.

The metabolism of fatty acids for the production of ATP occurs within mitochondria. This requires entry of activated fatty acids into the mitochondrial matrix, a process catalyzed by carnitine palmitoyl-transferase 1 (CPT1) and also requiring carnitine. CPT2 then removes carnitine from the fatty acid which can then enter the mitochondrial matrix for catabolism via beta-oxidation (McGarry and Brown, 1997).

Beta-oxidation appears to be important for oocyte nuclear maturation; meiotic maturation to metaphase II and extrusion of the first polar body (Downs et al., 2009; Valsangkar and Downs, 2013; Paczkowski et al., 2014). Further, it appears that betaoxidation is important in the acquisition of oocyte developmental competence (reviewed in McKeegan and Sturmey, 2011; Dunning et al., 2014b). Of particular interest here are studies demonstrating a reduction in beta-oxidation in both the oocyte and cumulus cell compartments of the COC when maturation occurs in vitro (Lee et al., 2011; Dunning et al., 2014a), demonstrating the importance of the ovarian follicular environment in appropriate regulation and/or the supply of metabolic factors that support this metabolic pathway (including supply of carnitine). The expression of genes involved in the beta-oxidation pathway are dysregulated in non-human primate cumulus cells and mouse COCs that have undergone maturation in vitro compared to in vivo maturation (Lee et al., 2011; Dunning et al., 2014a). In functional assays, both mouse and cat oocytes have decreased levels of beta-oxidation compared to those matured in vivo (Spindler et al. 2000; Dunning et al., 2014a). Specifically, we have shown that the rate of beta-oxidation in mouse COCs is significantly reduced, more than fifty percent, during IVM (Dunning et al., 2014a).

The importance of beta-oxidation is further exemplified in studies using inhibitors of this pathway during in vitro maturation. These studies demonstrate a requirement for beta-oxidation in nuclear maturation (Downs et al., 2009; Valsangkar and Downs, 2013; Paczkowski et al., 2014; Sanchez-Lazo et al., 2014), as described above, and in the acquisition of oocyte developmental competence in mouse, bovine and porcine oocytes (Ferguson and Leese, 2006; Sturmey et al., 2006; Dunning et al., 2010; Paczkowski et al., 2014).

We and others have shown that supplementation of IVM culture media with L-carnitine significantly increases beta-oxidation in mouse COCs (Dunning et al., 2010; Valsangkar and Downs, 2013). The inclusion of L-carnitine during maturation also results in a significant increase in oocytes reaching metaphase II in mouse (Dunning et al., 2011) and porcine (Somfai et al., 2011) and significantly more blastocyst embryos following fertilization (Dunning et al., 2010). The inclusion of L-carnitine has also been shown to increase oocyte mitochondrial activity in mouse (Wu et al., 2012), bovine (Hashimoto, 2009), and porcine (Somfai et al., 2011) and decrease intracellular lipid stores in porcine oocytes (Somfai et al., 2011). Further, our work has demonstrated the ability for L-carnitine to facilitate metabolism of intracellular lipid stores in preimplantation mouse and bovine embryos, significantly improving development (Dunning et al., 2010; Sutton-McDowall et al., 2012). Collectively, these studies illustrate the ability of Lcarnitine to upregulate beta-oxidation during IVM and utilize intracellular lipid stores. The beneficial effects of L-carnitine supplementation on oocyte quality can at least be partially ascribed to its effects on stimulating fatty acid beta-oxidation. The role of supplying exogenous fatty acid to IVM for utilization via betaoxidation, at least in the mouse, appears detrimental (Paczkowski et al., 2014). This may be due to the concentration or type of fatty acid used in this study. Elucidation of the oocytes preferential fatty acid type(s) and concentration and the detrimental effects of excess lipid in relation to oocyte developmental competence, is ongoing in a number of models and agriculturally important species (reviewed in Dunning et al., 2014b).

\section{Anti-oxidant capacity of L-carnitine during IVM}

Reactive oxygen species (ROS) are naturally formed during cellular metabolism, but kept in check by enzymatic and non-enzymatic antioxidants. If the production of ROS outweighs the cells antioxidant capacity this leads to oxidative stress (cellular damage via lipid peroxidation, DNA and protein damage). The process of in vitro culture itself generates increased oxidative stress, in addition to that generated from cellular metabolism, by exposure of cells to light, increased oxygen concentration and atmospheric conditions during handling (reviewed in Combelles et al., 2009). Specifically, in vitro maturation of oocytes has been shown to result in excessive production of ROS compared to in vivo matured oocytes (Banwell et al., 2007; Zeng et al., 2014). Further, the in vitro matured oocytes may be less equipped to attenuate deleterious effects of increased ROS compared to those matured in vivo (Uppangala et al., 2015). Collectively, this may contribute to the poorer developmental competence of IVM derived oocytes.

In vitro assays have demonstrated the ability of L-carnitine to act as an effective scavenger of superoxide anions, hydrogen peroxide and inhibit lipid peroxidation (Gulcin, 2006). The anti-oxidant property of L-carnitine is supported by in vitro studies demonstrating its capacity to prevent oxidant injury in various somatic cells (Silva-Adaya et al., 2008; Ribas et al., 2010). Thus, investigation of L-carnitines capacity to scavenge free radicles during IVM and its resultant effect on oocyte quality warrants investigation.

There are a number of studies demonstrating a link between inclusion of L-carnitine during IVM on reduced intracellular ROS and increased antioxidant enzymes within the oocyte of a number of species including mouse (Zare et al., 2015), bovine (Sovernigo et al., 2017), porcine (Somfai et al., 2011; You et al., 2012) and sheep (Mishra et al., 2016b). IVM of mouse oocytes in the presence of carnitine decreased ROS and increased the antioxidant glutathione (GSH) within 
oocytes and was associated with significantly increased rates of maturation to MII, cleavage and blastocyst embryos, but no change in blastocyst cell number (Zare et al., 2015). Similarly, the inclusion of L-carnitine during bovine IVM, in addition to reduced oxidative stress, resulted in significantly more blastocyst embryos with these embryos containing significantly increased number of cells (Sovernigo et al., 2017). Decreased ROS and increased GSH within porcine oocytes matured with L-carnitine also resulted in significantly improved oocyte developmental competence with increased rates of blastocysts following parthenogenetic activation (Wu et al., 2011; You et al., 2012) or somatic cell nuclear transfer (You et al., 2012).

Challenging the in vitro maturation environment by inclusion of hydrogen peroxide $\left(\mathrm{H}_{2} \mathrm{O}_{2}\right)$ or TNF $\alpha$ during oocyte maturation in vitro leads to a significant reduction in the number of mature oocytes and the number of blastocyst embryos post fertilization in both sheep and pig (Yazaki et al., 2013, Mishra et al., $2016 \mathrm{a}, \mathrm{b})$. The inclusion of L-carnitine has shown to reverse the effects of these exogenous insults on nuclear maturation (Yazaki et al., 2013; Mishra et al., 2016a) and capacity of the fertilized zygote to develop to blastocyst (Yazaki et al., 2013; Mishra et al., 2016a, b) The ability of L-carnitine to attenuate the detrimental effects of $\mathrm{H}_{2} \mathrm{O}_{2}$ during IVM has been shown to be associated with a reduction in ROS and increase in intracellular GSH within oocytes (Mishra et al., 2016b). To investigate the impact of increased ROS in human follicular fluid on oocyte quality following maturation, one study matured mouse oocytes in the presence of control follicular fluid and follicular fluid from women with endometriosis and suffering infertility (Giorgi et al., 2016). Oocyte maturation in the presence of follicular fluid from women with endometriosis resulted in a significant increase in meiotic spindle abnormalities that were reversed to control levels when $0.6 \mathrm{mg} / \mathrm{ml} \mathrm{L}$ carnitine was included in the maturation media (Giorgi et al., 2016).

Decreased oxidative stress and improved oocyte developmental competence in response to Lcarnitine has been associated with increased expression of cell cycle machinery in mouse oocytes (Zare et al., 2015); however the activity of these factors are regulated at the level of phosphorylation which was not investigated, likely due to the number of oocytes required to perform such experiments being prohibitive. A direct causal link between L-carnitine treatment and cell cycle machinery via carnitine's anti-oxidant capacity seems unlikely. Similarly, in porcine oocytes decreased ROS and improved developmental competence was associated with increased expression of genes involved in nuclear reprogramming (You et al., 2012) but again, a causal link between these pathways was not determined.

\section{Effects of L-carnitine during IVM on the cryotolerance of oocytes}

Oocyte cryopreservation would preserve fertility for women undergoing treatment for cancer and be useful in avoiding the effects of aging by preserving oocytes earlier in life. While human and mouse MII oocytes have been successfully used following cryopreservation, MII oocytes are susceptible to cryoinjury including spindle damage (Gomes et al., 2008; Huang et al., 2008). Cryopreservation of GV oocytes would be advantageous as they are not susceptible to damage inflicted by cryopreservation due to the chromosomes being at interphase, and utilizing oocytes at this stage would avoid the need for ovarian hyperstimulation and its associated risks. However, cryopreserved GV oocytes are developmentally compromised with significantly reduced number of blastocysts post-thawing and fertilization (Aono et al., 2003, 2005). Cryopreservation of oocytes in agriculturally important species also has advantages with the ability to bank oocytes from genetically important animals. However, cryopreserved bovine oocytes have poorer developmental competence on thawing. Supplementation of IVM and/or vitrification media with L-carnitine has resulted improvements in oocyte quality in mouse GV oocytes (Moawad et al., 2013, 2014) and bovine MII oocytes following cryopreservation and thawing (Spricigo et al., 2017). Specifically, the inclusion of L-carnitine during IVM and vitrification of mouse $\mathrm{GV}$ oocytes significantly increased the number of blastocyst embryos, comparable to the number of blastocysts developed from oocytes that were not vitrified or matured in vivo and vitrified (Moawad et al., 2013, 2014). L-carnitine treatment during IVM and vitrification was associated with improved meiotic spindle formation and distribution and increased activity of mitochondria (Moawad et al., 2014).

Conflicting evidence is seen in studies investigating the effects of L-carnitine on bovine oocyte developmental competence post vitrification/thawing. Lcarnitine inclusion during bovine IVM has been shown to result in significantly increased numbers of blastocysts following cryopreservation and thawing of oocytes, which was associated with redistribution of lipid within oocytes (Chankitisakul et al., 2013). In contrast, Phongnimitr et al. (2013) showed that Lcarnitine supplementation during IVM significantly improved subsequent development to blastocyst, but this effect was lost following vitrification. Similarly, inclusion of L-carnitine during IVM of prepubertal bovine oocytes had no beneficial effect on oocyte developmental competence post thawing (Spricigo et al., 2017). Thus, further research is required to determine the effects of L-carnitine on bovine oocyte cryotolerance.

\section{Summary and future directions}

There is mounting evidence in the literature that L-carnitine has beneficial effects during the in vitro maturation of oocytes. The mechanism by which Lcarnitine improves oocyte quality is likely to be via both increased beta-oxidation and reducing oxidative stress via is ability to scavenge free radicals. Future studies could interrogate the relative contribution of these 
actions to improved oocyte developmental competence.

Differential effects of L-carnitine in various studies may be a result of different animal strains/breeds used and different laboratory practices, including culture media composition or the use of fully grown oocytes or oocytes collected from smaller follicles or prepubertal animals.

Based on the studies discussed here, future improvements in IVM culture media may benefit from the inclusion of L-carnitine. However, as promotion of fatty acid beta-oxidation impacts glucose metabolism in the $\mathrm{COC}$, it is important to consider the appropriate balance between these metabolic pathways (Paczkowski et al., 2014), and utilization of in vivo maturation as the gold standard would assist in optimization.

As yet, there are no studies demonstrating impact of L-carnitine during IVM on offspring health. Use of L-carnitine during mouse preimplantation embryo development in vitro demonstrates safety (Truong et al., 2016). However, the effect of L-carnitine supplementation specifically during IVM on offspring health is yet to be studied in any species. Demonstration of safety in several mouse strains and animal models would be advisable before implementation in the fertility clinic.

\section{References}

Aono N, Naganuma T, Abe Y, Hara K, Sasada H, Sato E, Yoshida H. 2003. Successful production of blastocysts following ultrarapid vitrification with stepwise equilibriation of germinal vesicle-stage mouse oocytes. J Reprod Dev, 49:501-506.

Aono N, Abe Y, Hara K, Sasada H, Sato E, Yoshida H. 2005. Production of live offspring from mouse germinal vesicle-stage oocytes vitrified by a modified stepwise method, SWEID. Fertil Steril, 84(suppl. 2):1078-1082.

Banwell KM, Lane M, Russell DL, Kind KL, Thompson JG. 2007. Oxygen concentration during mouse oocyte in vitro maturation affects embryo and fetal development. Hum Reprod, 22:2768-2775.

Bremer J. 1983. Carnitine--metabolism and functions. Physiol Rev, 63:1420-1480.

Brown HM, Dunning KR, Sutton-McDowall M, Gilchrist RB, Thompson JG, Russell DL. 2017. Failure to launch: aberrant cumulus gene expression during oocyte in vitro maturation. Reproduction, 153:R109-R120.

Buckett WM, Chian RC, Dean NL, Sylvestre C, Holzer HE, Tan SL. 2008. Pregnancy loss in pregnancies conceived after in vitro oocyte maturation, conventional in vitro fertilization, and intracytoplasmic sperm injection. Fertil Steril, 90:546-550.

Chankitisakul V, Somfai T, Inaba Y, Techakumphu M, Nagai T. 2013. Supplementation of maturation medium with L-carnitine improves cryo-tolerance of bovine in vitro matured oocytes. Theriogenology, 79:590-598.

Combelles CM, Gupta S, Agarwal A. 2009. Could oxidative stress influence the in-vitro maturation of oocytes? Reprod Biomed Online, 18:864-880.
Downs SM, Mosey JL, Klinger J. 2009. Fatty acid oxidation and meiotic resumption in mouse oocytes. Mol Reprod Dev, 76:844-853.

Dunning KR, Cashman K, Russell DL, Thompson JG, Norman RJ, Robker RL. 2010. Beta-oxidation is essential for mouse oocyte developmental competence and early embryo development. Biol Reprod, 83:909918.

Dunning KR, Akison LK, Russell DL, Norman RJ, Robker RL. 2011. Increased beta-oxidation and improved oocyte developmental competence in response to L-carnitine during ovarian in vitro follicle development in mice. Biol Reprod, 85:548-555.

Dunning KR, Robker RL. 2012. Promoting lipid utilization with l-carnitine to improve oocyte quality. Anim Reprod Sci, 134:69-75.

Dunning KR, Anastasi MR, Zhang VJ, Russell DL, Robker RL. 2014a. Regulation of fatty acid oxidation in mouse cumulus-oocyte complexes during maturation and modulation by PPAR agonists. PloS One, 9:e87327. Dunning KR, Russell DL, Robker RL. 2014b. Lipids and oocyte developmental competence: the role of fatty acids and beta-oxidation. Reproduction, 148:R15-27.

Ferguson EM, Leese HJ. 2006. A potential role for triglyceride as an energy source during bovine oocyte maturation and early embryo development. Mol Reprod Dev, 73:1195-1201.

Gilchrist RB, Thompson JG. 2007. Oocyte maturation: emerging concepts and technologies to improve developmental potential in vitro. Theriogenology, 67:6-15.

Giorgi VS, Da Broi MG, Paz CC, Ferriani RA, Navarro PA. 2016. N-acetyl-cysteine and L-carnitine prevent meiotic oocyte damage induced by follicular fluid from infertile women with mild endometriosis. Reprod Sci, 23:342-351.

Gomes CM, Silva CA, Acevedo N, Baracat E, Serafini P, Smith GD. 2008. Influence of vitrification on mouse metaphase II oocyte spindle dynamics and chromatin alignment. Fertil Steril, 90:1396-1404.

Gulcin I. 2006. Antioxidant and antiradical activities of L-carnitine. Life Sci, 78:803-811.

Hashimoto S. 2009. Application of in vitro maturation to assisted reproductive technology. J Reprod Dev, 55:1-10.

Huang JY, Chen HY, Park JY, Tan SL, Chian RC. 2008. Comparison of spindle and chromosome configuration in in vitro- and in vivo-matured mouse oocytes after vitrification. Fertil Steril, 90:1424-1432.

Huynh FK, Green MF, Koves TR, Hirschey MD. 2014. Measurement of fatty acid oxidation rates in animal tissues and cell lines. Methods Enzymol, 542:391-405.

Kolodziejczyk J, Saluk-Juszczak J, Wachowicz B. 2011. L-Carnitine protects plasma components against oxidative alterations. Nutrition, 27:693-699.

Lane M, Robker RL, Robertson SA. 2014. Parenting from before conception. Science, 345:756-760.

Lee YS, VandeVoort CA, Gaughan JP, Midic U, Obradovic Z, Latham KE. 2011. Extensive effects of in vitro oocyte maturation on rhesus monkey cumulus cell transcriptome. Am J Physiol Endocrinol Metab, 


\section{1:E196-209.}

McGarry JD, Brown NF. 1997. The mitochondrial carnitine palmitoyltransferase system. From concept to molecular analysis. Eur J Biochem, 244:1-14.

McKeegan PJ, Sturmey RG. 2011. The role of fatty acids in oocyte and early embryo development. Reprod Fertil Dev, 24:59-67.

Mishra A, Reddy IJ, Gupta P, Mondal S. 2016a. Developmental regulation and modulation of apoptotic genes expression in sheep oocytes and embryos cultured in vitro with L-carnitine. Reprod Domest Anim, 51:1020-1029.

Mishra A, Reddy IJ, Gupta PS, Mondal S. 2016b. Lcarnitine mediated reduction in oxidative stress and alteration in transcript level of antioxidant enzymes in sheep embryos produced in vitro. Reprod Domest Anim, 51:311-321.

Moawad AR, Tan SL, Xu B, Chen HY, Taketo T. 2013. L-carnitine supplementation during vitrification of mouse oocytes at the germinal vesicle stage improves preimplantation development following maturation and fertilization in vitro. Biol Reprod, 88:104. doi 10.1095/biolreprod.112.107433.

Moawad AR, Xu B, Tan SL, Taketo T. 2014. Lcarnitine supplementation during vitrification of mouse germinal vesicle stage-oocytes and their subsequent in vitro maturation improves meiotic spindle configuration and mitochondrial distribution in metaphase II oocytes. Hum Reprod, 29:2256-2268.

Montjean D, Entezami F, Lichtblau I, Belloc S, Gurgan T, Menezo Y. 2012. Carnitine content in the follicular fluid and expression of the enzymes involved in beta oxidation in oocytes and cumulus cells. J Assist Reprod Genet, 29:1221-1225.

Nada MA, Rhead WJ, Sprecher H, Schulz H, Roe CR. 1995. Evidence for intermediate channeling in mitochondrial beta-oxidation. J Biol Chem, 270:530535

Paczkowski M, Schoolcraft WB, Krisher RL. 2014. Fatty acid metabolism during maturation affects glucose uptake and is essential to oocyte competence. Reproduction, 148:429-439.

Phongnimitr T, Liang Y, Srirattana K, Panyawai K, Sripunya N, Treetampinich C, Parnpai R. 2013 Effect of L-carnitine on maturation, cryo-tolerance and embryo developmental competence of bovine oocytes. Anim Sci J, 84:719-725.

Reader KL, Cox NR, Stanton JA, Juengel JL. 2015. Effects of acetyl-L-carnitine on lamb oocyte blastocyst rate, ultrastructure, and mitochondrial DNA copy number. Theriogenology, 83:1484-1492.

Rebouche CJ. 1992. Carnitine function and requirements during the life cycle. FASEB J, 6:33793386

Ribas GS, Manfredini V, de Marco MG, Vieira RB, Wayhs CY, Vanzin CS, Biancini GB, Wajner M, Vargas CR. 2010. Prevention by L-carnitine of DNA damage induced by propionic and L-methylmalonic acids in human peripheral leukocytes in vitro. Mutat Res, 702:123-128.

Rizos D, Ward F, Duffy P, Boland MP, Lonergan $P$. 2002. Consequences of bovine oocyte maturation, fertilization or early embryo development in vitro versus in vivo: implications for blastocyst yield and blastocyst quality. Mol Reprod Dev, 61:234-248.

Sanchez-Lazo L, Brisard D, Elis S, Maillard V, Uzbekov R, Labas V, Desmarchais A, Papillier $\mathbf{P}$, Monget P, Uzbekova S. 2014. Fatty acid synthesis and oxidation in cumulus cells support oocyte maturation in bovine. Mol Endocrinol, 28:1502-1521.

Sener G, Paskaloglu K, Satiroglu H, Alican I, Kacmaz A, Sakarcan A. 2004. L-carnitine ameliorates oxidative damage due to chronic renal failure in rats. $J$ Cardiovasc Pharmacol, 43:698-705.

Silva-Adaya D, Perez-De La Cruz V, HerreraMundo MN, Mendoza-Macedo K, VilledaHernandez J, Binienda Z, Ali SF, Santamaria A. 2008. Excitotoxic damage, disrupted energy metabolism, and oxidative stress in the rat brain: antioxidant and neuroprotective effects of L-carnitine. $J$ Neurochem, 105:677-689.

Somfai T, Kaneda M, Akagi S, Watanabe S, Haraguchi S, Mizutani E, Dang-Nguyen TQ, Geshi M, Kikuchi K, Nagai T. 2011. Enhancement of lipid metabolism with L-carnitine during in vitro maturation improves nuclear maturation and cleavage ability of follicular porcine oocytes. Reprod Fertil Dev, 23:912920.

Sovernigo TC, Adona PR, Monzani PS, Guemra S, Barros F, Lopes FG, Leal C. 2017. Effects of supplementation of medium with different antioxidants during in vitro maturation of bovine oocytes on subsequent embryo production. Reprod Domest Anim. doi: 10.1111/rda.12946.

Spindler RE, Pukazhenthi BS, Wildt DE. 2000. Oocyte metabolism predicts the development of cat embryos to blastocyst in vitro. Mol Reprod Dev, 56:163171.

Spricigo JF, Morato R, Arcarons N, Yeste M, Dode MA, Lopez-Bejar M, Mogas T. 2017. Assessment of the effect of adding L-carnitine and/or resveratrol to maturation medium before vitrification on in vitromatured calf oocytes. Theriogenology, 89:47-57.

Sturmey RG, O'Toole PJ, Leese HJ. 2006. Fluorescence resonance energy transfer analysis of mitochondrial:lipid association in the porcine oocyte. Reproduction, 132:829-837.

Sutton ML, Gilchrist RB, Thompson JG. 2003. Effects of in-vivo and in-vitro environments on the metabolism of the cumulus-oocyte complex and its influence on oocyte developmental capacity. Hum Reprod Update, 9:35-48.

Sutton-McDowall ML, Feil D, Robker RL, Thompson JG, Dunning KR. 2012. Utilization of endogenous fatty acid stores for energy production in bovine preimplantation embryos. Theriogenology, 77:1632-1641.

Truong TT, Soh YM, Gardner DK. 2016. Antioxidants improve mouse preimplantation embryo development and viability. Hum Reprod, 31:1445-1454. Uppangala S, Dhiman S, Salian SR, Singh VJ, Kalthur G, Adiga SK. 2015. In vitro matured oocytes are more susceptible than in vivo matured oocytes to mock ICSI induced functional and genetic changes. 
PloS One, 10:e0119735.

Valckx SD, De Pauw I, De Neubourg D, Inion I, Berth M, Fransen E, Bols PE, Leroy JL. 2012. BMIrelated metabolic composition of the follicular fluid of women undergoing assisted reproductive treatment and the consequences for oocyte and embryo quality. Hum Reprod, 27:3531-3539.

Valsangkar D, Downs SM. 2013. A requirement for fatty acid oxidation in the hormone-induced meiotic maturation of mouse oocytes. Biol Reprod, 89:43. doi: 10.1095/biolreprod.113.109058.

Van Blerkom J, Davis PW, Lee J. 1995. ATP content of human oocytes and developmental potential and outcome after in-vitro fertilization and embryo transfer. Hum Reprod, 10:415-424.

Varnagy A, Bene J, Sulyok E, Kovacs GL, Bodis J, Melegh B. 2013. Acylcarnitine esters profiling of serum and follicular fluid in patients undergoing in vitro fertilization. Reprod Biol Endocrinol, 11:67. doi: 10.1186/1477-7827-11-67.

Vaz FM, Wanders RJ. 2002. Carnitine biosynthesis in mammals. Biochem J, 361:417-429.

Wu GQ, Jia BY, Li JJ, Fu XW, Zhou GB, Hou YP, Zhu SE. 2011. L-carnitine enhances oocyte maturation and development of parthenogenetic embryos in pigs. Theriogenology, 76:785-793.

Wu LL, Russell DL, Norman RJ, Robker RL. 2012. Endoplasmic reticulum (ER) stress in cumulus-oocyte complexes impairs pentraxin-3 secretion, mitochondrial membrane potential (DeltaPsi $\mathrm{m}$ ), and embryo development. Mol Endocrinol, 26:562-573.
Yazaki T, Hiradate Y, Hoshino Y, Tanemura K, Sato E. 2013. L-carnitine improves hydrogen peroxideinduced impairment of nuclear maturation in porcine oocytes. Anim Sci J, 84:395-402.

Ye J, Li J, Yu Y, Wei Q, Deng W, Yu L. 2010. Lcarnitine attenuates oxidant injury in HK-2 cells via ROS-mitochondria pathway. Regul Pept, 161:58-66.

You J, Lee J, Hyun SH, Lee E. 2012. L-carnitine treatment during oocyte maturation improves in vitro development of cloned pig embryos by influencing intracellular glutathione synthesis and embryonic gene expression. Theriogenology, 78:235-243.

Zare Z, Masteri Farahani R, Salehi M, Piryaei A, Ghaffari Novin M, Fadaei Fathabadi F, Mohammadi M, Dehghani-Mohammadabadi M. 2015. Effect of Lcarnitine supplementation on maturation and early embryo development of immature mouse oocytes selected by brilliant cresyle blue staining. J Assist Reprod Genet, 32:635-643.

Zeng HT, Richani D, Sutton-McDowall ML, Ren Z, Smitz JE, Stokes Y, Gilchrist RB, Thompson JG. 2014. Prematuration with cyclic adenosine monophosphate modulators alters cumulus cell and oocyte metabolism and enhances developmental competence of in vitro-matured mouse oocytes. Biol Reprod, 91:47.

Zhao H, Zhao Y, Li T, Li M, Li J, Li R, Liu P, Yu Y, Qiao J. 2015. Metabolism alteration in follicular niche: The nexus among intermediary metabolism, mitochondrial function, and classic polycystic ovary syndrome. Free Radic Biol Med, 86:295-307. 